Japan. J. Med. Sci. Biol., 33, 189-201, 1980

\title{
INACTIVATION AND REACTIVATION OF 20-HYDROXYECDYSONE DURING PUPAL-ADULT DEVELOPMENT OF THE FLESHFLY, SARCOPHAGA PEREGRINA
}

\author{
Atsuko MORIBAyASHI and Tetsuya OHTAKI \\ Laboratory of Technology, National Institute of Health, Shinagawa-ku, \\ Tokyo 141, and Department of Biology, Faculty of Science, \\ Kanazawa University, 1-1 Marunouchi, Kanazawa 920
}

(Received, April 4, 1980. Accepted, June 10, 1980)

\begin{abstract}
SUMMARY: The inactivation process of ecdysteroids during pupal-adult development of the fleshfly, Sarcophaga peregrina, was analyzed by following injected ${ }^{3} \mathbf{H}$ 20-hydroxyecdysone. 20-Hydroxyecdysone was rapidly metabolized and converted mostly into three groups of metabolites, one of which was closely related to 20 . hydroxyecdysone in its chemical nature and the other two were polar conjugates of 20-hydroxyecdysone (OA and $\mathrm{OB}$ ). The first group of metabolites decreased through the first day after injection of 20-hydroxyecdysone, and began to increase thereafter. Those of conjugates $\mathrm{OA}$ and $\mathrm{OB}$ increased through the first day after the injection and then began to decrease. The conjugate $\mathrm{OA}$ introduced into phraate pupae changed to less polar substances, liberating 20-hydroxyecdysone at the beginning of adult development. Since the conjugates $\mathrm{OA}$ and $\mathrm{OB}$ did not exhibit any appreciable moulting hormone activity, we consider that some part of 20-hydroxyecdysone synthesized during larval-pupal development is stored as conjugate forms until the beginning of adult development, and that the conjugate $\mathrm{OA}$ is converted to 20 hydroxyecdysone for adult development.
\end{abstract}

\section{INTRODUCTION}

The ecdysteroid content of the whole body of the insect varies with the developmental stage from mature larvae to pupae. In the study on the ecdysteroid titer from immature larvae to the adult of a blowfly, Shaaya and Karlsone (1965) found two maxima for that stages; one arose about $6 \mathrm{hr}$ after pupariation and the other two days after pupation.

In Sarcophaga larvae, the ecdysteroid titer began to increase after they were transferred to the dry condition and reached a maximum $12 \mathrm{hr}$ after pupariation (Ohtaki and Takahashi, 1972). About $40 \mathrm{hr}$ after pupariation, the ecdysteroid titer decreased to a minimum value. Afterwards, only in nondiapause pupae, the titer began to increase again at the time of the initiation of adult development. The first maximum of the ecdysteroid titer is thought to be responsible

森林敦子 (国立予防衛生研究所技術部)

大滝哲也（金沢大学理学部金沢市丸の内 1-1) 
for larval-pupal development and the second peak for adult development, since the second one was not seen in diapause pupae.

In the previous paper (Moribayashi and Ohtaki, 1978), we described three different pathways of inactivation of 20-hydroxyecdysone in larval-pupal development of Sarcophaga peregrina; (1) catabolic degradation, (2) formation of the conjugates, and (3) epimerization to 3-epi-20-hydroxyecdysone. We found also that the conjugated 20-hydroxyecdysteroids could be separated into two components, the conjugate $O A$ and the conjugate $O B$, which differed from each other chemically in their behavior on TLC and susceptibility to glusulase. In this study, we further examined the fate of 20-hydroxyecdysone metabolites in pupal-adult development of $S$. peregrina.

\section{Materials AND METHODS}

Animals and injections: Twenty hours after puparium formation at $25 \mathrm{C}$, the fleshfly $S$. peregrina was used as "pharate pupa" in these experiments. Pharate pupae were given $1 \mu \mathrm{g}$ of ${ }^{3} \mathrm{H}$-20-hydroxyecdysone $(0.025 \mu \mathrm{Ci})$ in $1 \mu \mathrm{l}$ of water to determine the biological activity of ecdysteroid metabolites and to obtain the conjugated 20-hydroxyecdysteroids, $\mathrm{OA}$ and $\mathrm{OB}$, or $0.2 \mu \mathrm{g}$ of ${ }^{3} \mathrm{H}-20$-hydroxyecdysone $(0.1 \mu \mathrm{Ci})$ in $1 \mu \mathrm{l}$ of water to follow the fate of each metabolite. Physiological amounts of $\mathrm{OA}$ and $\mathrm{OB}$ were injected into pharate pupa and pupa at each stage.

Chemicals: Radioactive ${ }^{3} \mathrm{H}-20$-hydroxyecdysone (specific activity $3.27 \mathrm{Ci} / \mathrm{m}$ mol) and 20-hydroxyecdysone were purchased from New England Nuclear Co., Boston, and from Rohto Pharmaceutical Co., Osaka, respectively. Insta-gel, a universal liquid scintillation cocktal, was obtained from Packard Instrument Company, Inc. Glusulase containing sulfatase and glucuronidase was purchased from Endo Laboratories, Inc., New York. The standard sample for high performance liquid chromatography (HPLC), 3-epi-20-hydroxyecdysone, was kindly provided by Dr. Ikekawa, Tokyo Institute of Technology.

Extraction of ecdysteroids: Pupae were homogenized in acetone:ethanol ( $1: 1$ by vol.) and ecdysteroids were extracted several times from the homogenates. with the same solvent. The combined extracts were evaporated under reduced pressure and the residue was dissolved in methanol. After centrifugation of the methanol solution at $4,000 \mathrm{rpm}$ for $10 \mathrm{~min}$, the supernatant was concentrated, and the residue was used for measurement of the radioactivity. For purification, the residue was applied onto an amberlite XAD- 4 column, and was eluted with water and subsequently with methanol. At least $95 \%$ of the radioactivity applied on the column was recovered in the fraction eluted with methanol. The methanol fraction was subjected to thin layer chromatography (TLC).

Thin layer chromatography (TLC): 20-Hydroxyecdysone and its metabolites were separated on silica gel TL plates (Merck Darmstadat, $0.25 \mathrm{~mm}$ thick, with fluorescent indicator) with the solvent system of either chloroform: methanol (4:1 by vol.) (system A) or ethylacetate:ethanol:water (2:8:1 by vol.) (system $\mathrm{B}$ ). 
Silica gel in 5-mm strips of the TL plates was scraped into scintillation vials. Methanol $(0.5 \mathrm{ml})$ and Insta-gel $(5 \mathrm{ml})$ were added, and the radioactivity was measured. Alternatively, the wide zone corresponding to 20-hydroxyecdysone on TL plates developed with solvent system A was scraped, and the absorbed materials were eluted with chloroform:methanol (1:1 by vol.). The eluted materials were subjected to high performance liquid chromatography (HPLC). The substance remaining at the origin on the TL plate developed with solvent system A was eluted with methanol. The methanol extract was hydrolyzed with glusulase in $0.5 \mathrm{M}$ acetate buffer ( $\mathrm{pH}$ 5.3) (Mizuno and Ohnishi, 1975; Moribayashi and Ohtaki, 1978). The substance treated with glusulase was again developed with solvent system $A$, and materials scraped from the wide zone corresponding to 20-hydroxyecdysone were again subjected to HPLC.

High performance liquid chromatography (HPLC): HPLC was used for determination of 20-hydroxyecdysone and closely related substances. A Zorbax SIL column ( $15 \mathrm{~cm}$ in length and $4.6 \mathrm{~mm}$ in inside diameter) was used. Fractionation was carried out under the following conditions; mobile phase, n-hexane:ethanol:methanol:acetonitrile (85:15:2:2 by vol.); flow rate $0.8 \mathrm{~m} / \mathrm{min}$; column pressure $25 \mathrm{~kg} / \mathrm{cm}^{2}$; and detector, an ultraviolet photometer at $254 \mathrm{~nm}$. The radioactivity of each fraction separated by HPLC was measured.

Bioassay: The moulting hormone activity in extracts was assayed by the methods described earlier (Ohtaki et al., 1968).

\section{RESULTS}

\section{Metabolic Fate of Injected 20-hydroxyecdysone Followed by TLC and HPLC Analyses}

Batches of about 30 "pharate pupae" were homogenized $6 \mathrm{hr}$ and one, 2 and 4 days after injection of ${ }^{3} \mathrm{H}-20$-hydroxyecdysone. Figure 1 (filled circles) shows the radioactivity recovered in each extract with methanol. During the initial $6 \mathrm{hr}$, about $65 \%$ of the injected radioactivity disappeared from whole extracts and then the disappearance became gradual. When each methanol extract was subjected to TLC with solvent system B (as described in Methods), the extracted substances were separated mostly into three components designated as OA, OB and the unconjugated ecdysteroids in the increasing order in the $\mathrm{Rf}$ value on a TL chromatogram (Fig. 2). The group of substances designated as the unconjugated ecdysteroids consists of 20-hydroxyecdysone itself and its metabolites whose chemical natures are closely related to 20-hydroxyecdysone. These substances were not separated by TLC with solvent system B. The unconjugated ecdysteroids containing 20-hydroxyecdysone decreased rapidly during the day after injection of 20-hydroxyecdysone and began to increase thereafter. However, $\mathrm{OA}$ and $\mathrm{OB}$ presumed to be the conjugated ecdysteroids conversely increased during the day after the injection, and then decreased gradually. These results suggest that the increased rate of the unconjugated ecdysteroids is due to the 


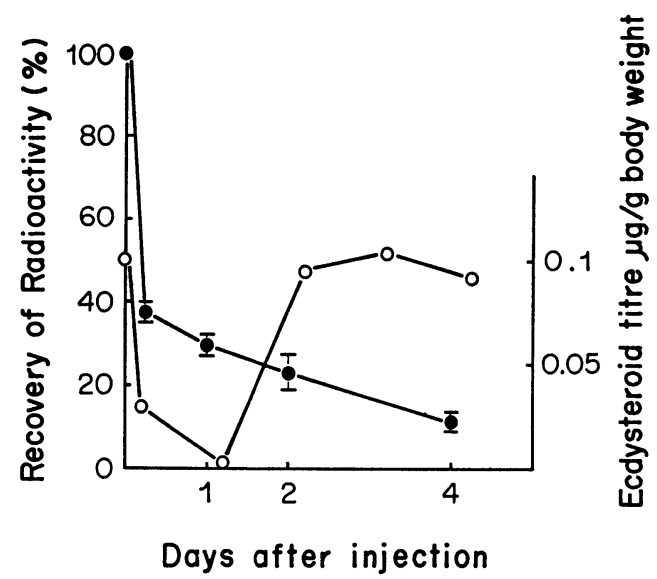

Fig. 1. Percentage of the radioactivity recovered $6 \mathrm{hr}$ and 1,2 and 4 days after injection of ${ }^{3} \mathrm{H}$-20-hydroxyecdysone into 20 -hr-old puparia of $S$. peregrina (filled circles). Bars indicate the upper and the lower limits of the standard error. For reference, the ecdysteroid titer of the larval body in nondiapause pupae (equivalent to 20hydroxyecdysone) is shown (Ohtaki and Takahashi, 1972) (open circles).

liberation of the unconjugated ecdysteroids from $\mathrm{OA}$ or $\mathrm{OB}$. The time when the amount of the unconjugated ecdysteroids increased corresponded to the stage of initiation of adult development in nondiapause pupae (Fig. 1, open circles and Fig. 2, open circles). For HPLC analysis, each methanol extract was developed with solvent system A (as described in Methods) on a TL plate, and the materials eluted from rather the wide region around the $\mathrm{Rf}$ value of 20-hydroxyecdysone were subjected to HPLC. The elution times of radioactive substances were compared with those of authentic standards. As shown in Fig. 3, 3-epi-20-hydroxyecdysone and unknown unconjugated ecdysteroids (peaks Nos. 1, 2 and 3) were detected besides 20-hydroxyecdysone. The radioactivities of 20-hydroxyecdysone in the extracts obtained from animals $6 \mathrm{hr}$ and one, 2 and 4 days after injection of 20-hydroxyecdysone into "pharate pupae" amounted to about $68 \%, 45 \%, 50 \%$ and $65 \%$ of the dose applied to HPLC, respectively.

The conjugates $\mathrm{OA}$ and $\mathrm{OB}$ were extracted with methanol from the origin on TL plates developed with system A. After treated with glusulase, the methanol extract was again developed with the same solvent. A considerable radioactivity was recovered from the area corresponding in the $\mathrm{Rf}$ value to that of 20-hydroxyecdysone. The less polar radioactive unconjugated ecdysteroids thus obtained were subjected to HPLC, and separated into several compounds, which were similar to those shown in Fig. 3, although 3-epi-20-hydroxyecdysone and the compound of peak number 3 were not clearly recognized. The radioactivities of 20-hydroxyecdysone in the conjugated ecdysteroids obtained from animals $6 \mathrm{hr}$, and one, 2 and 4 days after the injection into "pharate pupa" amounted to about $32 \%, 55 \%, 50 \%$ and $37 \%$ of the dose applied to HPLC, respectively. The percentages of 20 -hydroxyecdysone in the conjugated and the 


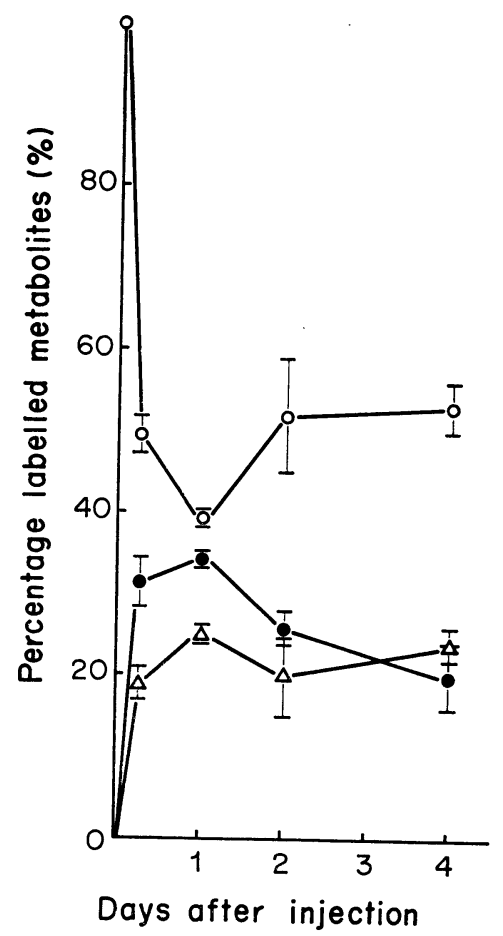

Fig. 2. TLC separation of the metabolites. Radioactive percentage of the three main compounds isolated from extracts $6 \mathrm{hr}$ and 1,2 and 4 days after injection of ${ }^{3} \mathrm{H}-20$ hydroxyecdysone into 20-hr-old puparia. They are named in the order of increasing Rf value; ecdysteroid conjugate $\mathrm{OA}(\triangle)$, ecdysteroid conjugate $\mathrm{OB}(\bullet)$ and substances closely related to 20-hydroxyecdysone, the unconjugated ecdysteroids (O). After chromatography with $\mathrm{CH}_{3} \mathrm{COOC}_{2} \mathrm{H}_{5}: \mathrm{C}_{2} \mathrm{H}_{5} \mathrm{OH}: \mathrm{H}_{2} \mathrm{O}$ (2:8:1 by vol.), silica gel on the chromatogram was scraped off in $5-\mathrm{mm}$ width. Radioactivity was measured in a scintillation counter. Bars indicate the upper and the lower limits of the standard error.

unconjugated ecdysteroids to the injected dose of 20-hydroxyecdysone are shown in Fig. 4. The unconjugated ecdysteroids of the compounds of peak numbers 1 , 2 and 3 recognized in HPLC have not been identified (Fig. 3).

\section{Metabolic Fate of the Radioactive Ecdysteroid Conjugates}

The metabolic fates of $\mathrm{OA}$ and $\mathrm{OB}$ in pharate pupae and pupae were studied by injecting physiological amounts of purified radioactive OA or OB into insects. OA and OB injected into "pharate pupae" were extracted in $6 \mathrm{hr}$ and one, 2 and 3 days. OB was metabolized faster than OA (Fig. 5). Difference in the metabolic rate between $\mathrm{OA}$ and $\mathrm{OB}$ was further examined in the three definite developmental stages; larval-pupal apolysis (0 day in Fig. 6), larvalpupal ecdysis (one day) and initiation of adult development (2 days). Figure 6 

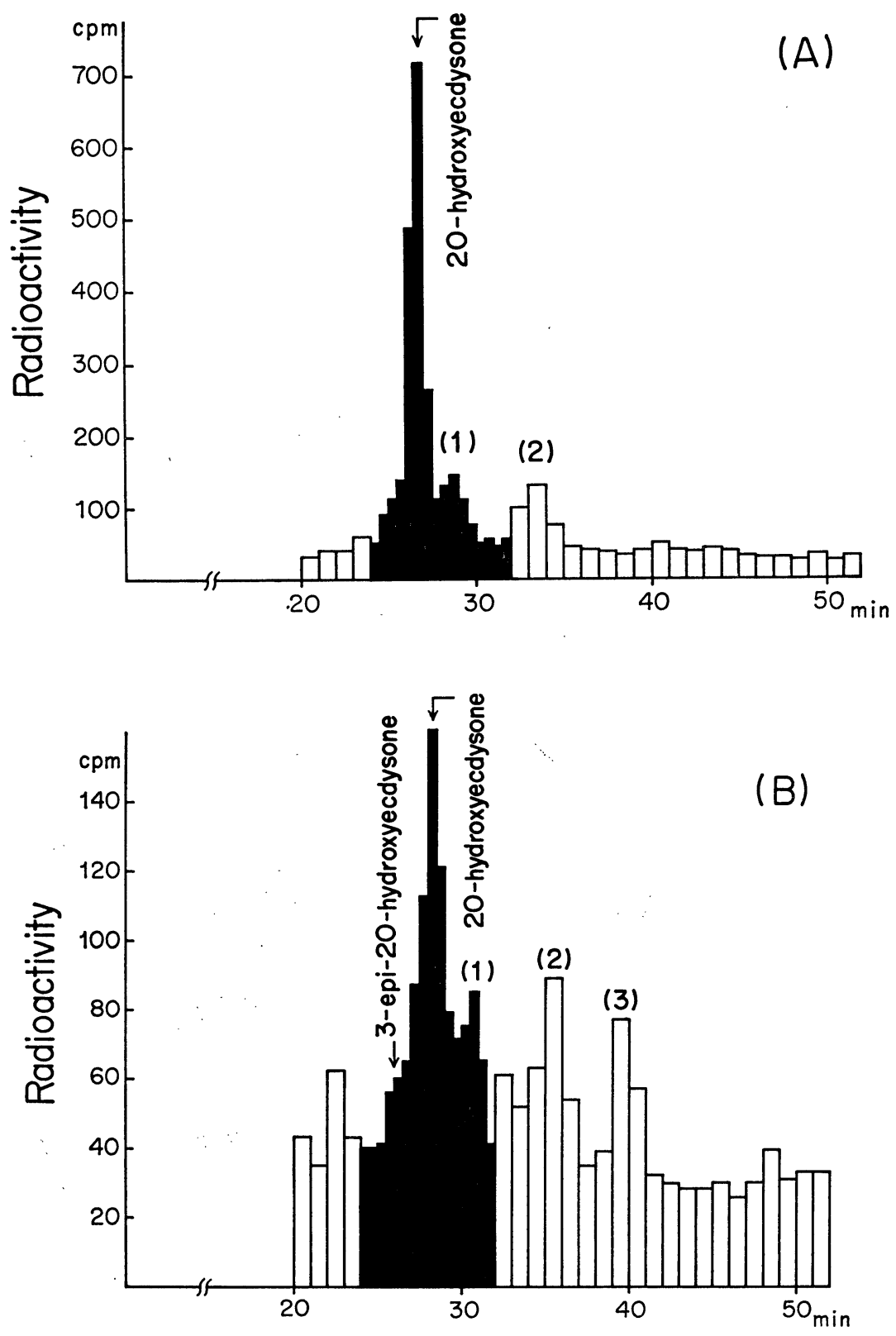

shows that disappearance of the injected radioactive $O A$ and $O B$ a day after larval-pupal apolysis, the stage at which formation of the ecdysteroid conjugates is predominant, was lower than that for other stages. Possible metabolites were extracted at different time intervals after injection of $O A$ or $O B$ for 


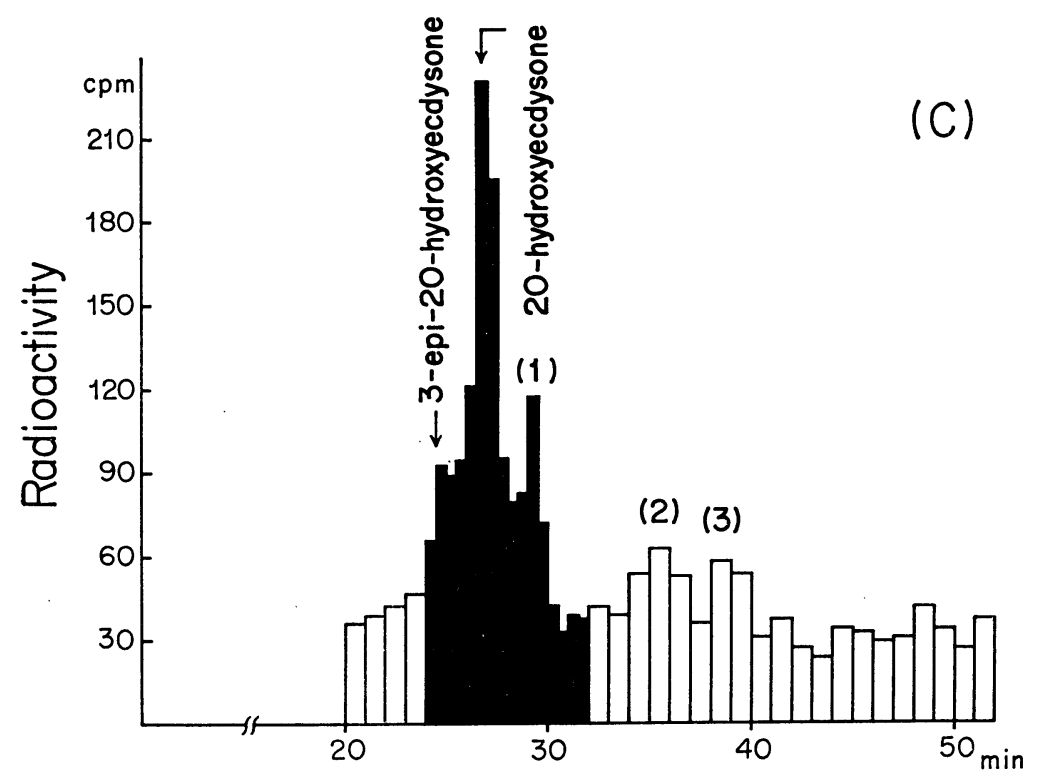

Fig. 3. Radiochromatograms of ecdysteroid metabolites in the fraction corresponding to 20-hydroxyecdysone eluted by HPLC. The extracts from pharate pupae $6 \mathrm{hr}(\mathrm{A})$, 1 day (B) and 4 days (C) after injection of ${ }^{3} \mathrm{H}$-20-hydroxyecdysone were subjected to HPLC. Outflows were collected every $30 \mathrm{sec}$ or $1 \mathrm{~min}$, and counted in a scintillation counter. The open bars and filled bars show cpm of samples collected every $1 \mathrm{~min}$ and every $30 \mathrm{sec}$, respectively. Peaks number (1), (2) and (3) are the unknown ecdysteroids more polar than 20-hydroxyecdysone.

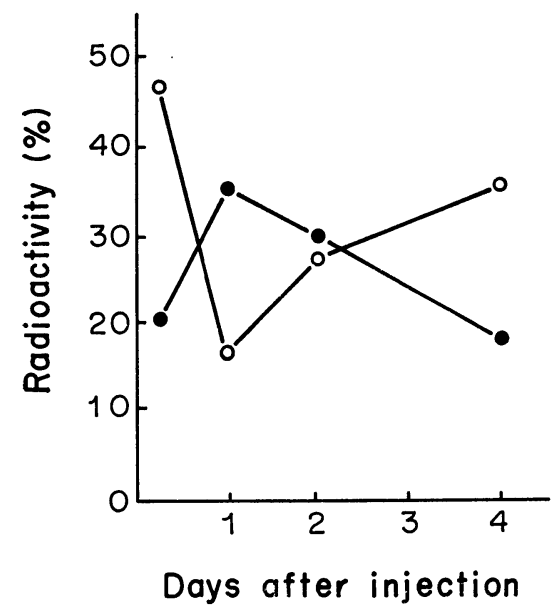

Fig. 4. 20-Hydroxyecdysone in the unconjugated ecdysteroids (open circles) and in the conjugated ecdysteroids (filled circles). Percent radioactivities to recovered unconjugate and conjugate are shown. For further explanation, see text. 


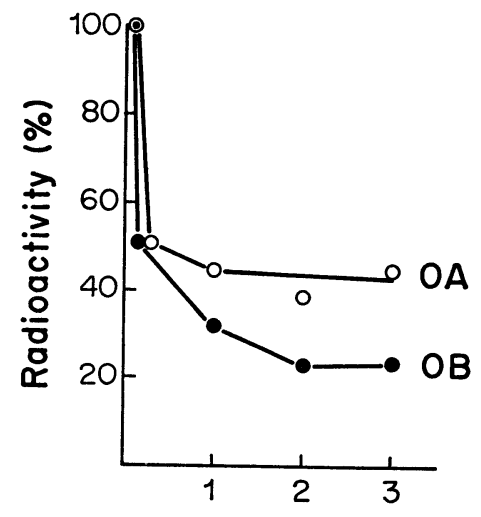

\section{Days after injection}

Fig. 5. Recoveries of conjugates $O A$ and $O B$ extracted $6 \mathrm{hr}$ and 1, 2 and 3 days after injection of $\mathrm{OA}$ and $\mathrm{OB}$, respectively. Percentages to the injected radioactivity are shown.

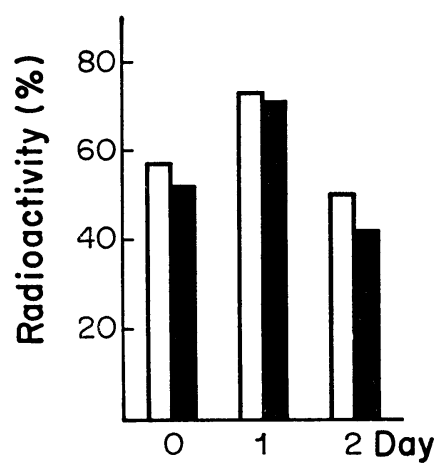

Fig. 6. Radioactivities recovered $3 \mathrm{hr}$ after administration of $\mathrm{OA}$ (open bar) and $\mathrm{OB}$ (filled bar) into 20-hr-old (day 0), 44-hr-old (day 1), and 68-hr-old (day 2) puparia, respectively. Percentages against the injected radioactivity are shown.

analyzing their chemical nature. The radioactive metabolites of $\mathrm{OA}$ and $\mathrm{OB}$ in each extract were separated by TLC with solvent system B. Chromatographic patterns of metabolites of conjugates $\mathrm{OA}$ and $\mathrm{OB}$ are shown in Figs. 7 and 8, respectively. The radioactive materials obtained by injection of $\mathrm{OA}$ were almost identical to those obtained by injection of 20 -hydroxyecdysone. The radioactive materials at peaks Nos. 1, 2, and 3 in Fig. 7 may be OA, OB and the unconjugated ecdysteroids, respectively. The rate of conversion from $\mathrm{OA}$ to $\mathrm{OB}$ or to the unconjugated ecdysteroids increased with the time after injection of OA. Whereas, injected OB was recovered without any detectable change (Fig. 8). From these results, we suppose that the increase of the unconjugated ecdysteroid is due to the release of the unconjugated ecdysteroids from conjugate OA. 


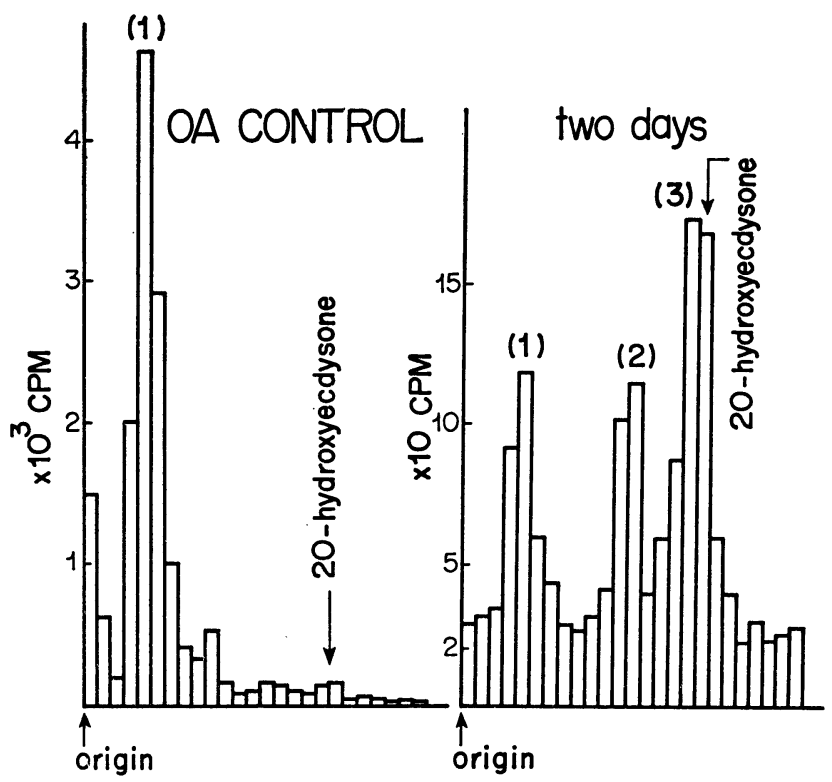

Fig. 7. Radiochromatograms of purified conjugate $\mathrm{OA}$ used in this study, and of extracts 2 days after injection of conjugate $\mathrm{OA}$. TL plate was developed with $\mathrm{CH}_{3} \mathrm{COOC}_{2} \mathrm{H}_{5}$ : $\mathrm{C}_{2} \mathrm{H}_{5} \mathrm{OH}: \mathrm{H}_{2} \mathrm{O}$ (2:8:1 by vol.). Peaks Nos. (1), (2) and (3) correspond to OA, OB, and the unconjugated ecdysteroids, respectively.

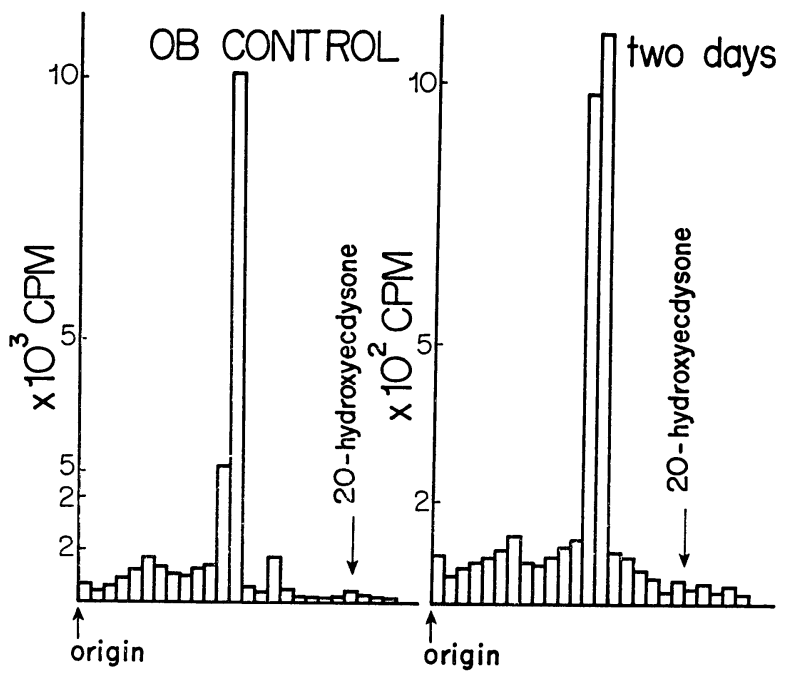

Fig. 8. Radiochromatograms of purified conjugate $O B$ used in this study and of extracts 2 days after injection of conjugate OB. Solvent system is the same as that in Fig. 7 . 
TABLE I

Relation between radioactivity and biological activity recovered from pharate pupae of S. peregrina treated with ${ }^{3} \mathrm{H}-20$-hydroxyecdysone

\begin{tabular}{lc|ccc}
\hline & $\begin{array}{c}\text { Radioactivity } \\
(\%)\end{array}$ & I $(\mu \mathrm{g})$ & II $(\mu \mathrm{g})$ & III $(\mathrm{II} / \mathrm{I} / 100)$ \\
Methanol extract & 33.5 & 0.0693 & 0.0160 & 23.1 \\
Unconjugated ecdysteroids & 15.0 & 0.0641 & 0.0320 & 50.0 \\
Conjugate OA & 4.0 & 0.0516 & 0.0012 & 2.4 \\
Conjugate OB & 11.2 & 0.1171 & 0.0020 & 1.7 \\
\hline
\end{tabular}

Each metabolite was extracted from pharate pupae $24 \mathrm{hr}$ after the injection of $1 \mu \mathrm{g}$ of ${ }^{3} \mathrm{H}-20-$ hydroxyecdysone in $1 \mu \mathrm{l}$ of water and further separated by TLC.

I: The amount of ecdysteroids injected into each test abdomen calculated from radioactivity.

II : The equivalent amount of 20-hydroxyecdysone calculated from biological activity.

III : Ratio (\%) of biological activity (II) to ecdysteroids (I).

\section{Biological Activity of Each Metabolite}

Biological activity of each ecdysteroid metabolite is shown in Table I. As described in Methods, the methanol extract was separated into three groups: the unconjugated ecdysteroids and conjugates $\mathrm{OA}$ and $\mathrm{OB}$. As is clearly shown in Table I, the biological activity was demonstrated only in the unconjugated ecdysteroids fraction. $\mathrm{OA}$ or $\mathrm{OB}$ did not show any appreciable biological activity. As shown in Fig. 4, the rate of 20-hydroxyecdysone in the unconjugated ecdysteroids showing the biological activity was minimum a day after injection of 20-hydroxyecdysone. The biological activity in one day was the lowest in the stage of pupal-adult development (Fig. 1, open circles). From these results, we deduced that all of the biological activity arose mainly from 20-hydroxyecdysone in the unconjugated ecdysteroids.

\section{Discussion}

It is generally recognized that the brain hormone stimulates the synthesis of ecdysone as a trigger of the transformation from larva to adult. However, the prothoracic gland dissected from the silkworm, Bombyx mori, $36 \mathrm{hr}$ after pupation scarcely retained this function of synthesizing ecdysone (Sakurai and Ohtaki, unpublished data).

In Sarcophaga peregrina, the ecdysteroid titer changes with development from larva to adult. There is virtually no difference in the titers between diapausing and nondiapausing pupae until larval-pupal ecdysis, which usually takes place $40 \mathrm{hr}$ after pupariation. Thereafter the ecdysteroid titer in diapausing pupae remained rather unchanged, but that in nondiapausing pupae increased dramatically at the time of adult development (Fig. 1, open circles, 


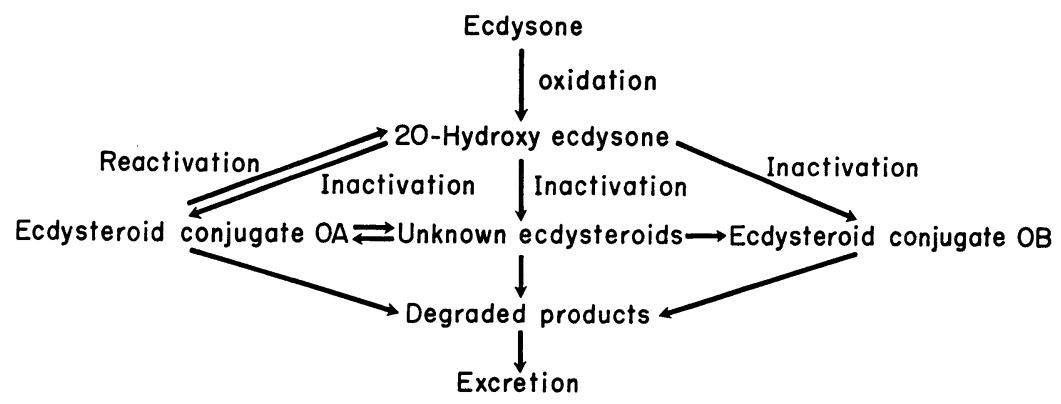

Fig. 9. A proposed scheme of the inactivation and the reactivation of 20-hydroxyecdysone in $S$. peregrina. For further explanation, see text.

Ohtaki and Takahashi, 1972). Therefore, we attempted to find out how the endocrinological and physiological mechanisms were involved in pupal-adult development. ${ }^{3} \mathrm{H}$-20-hydroxyecdysone injected into $S$. peregrina was metabolized and converted mostly into three groups of metabolites. The rate of conversion of 20-hydroxyecdysone to conjugate $\mathrm{OA}$ or $\mathrm{OB}$ became maximum one day after injection of 20-hydroxyecdysone and then decreased gradually. Conversely, the rate of the unconjugated ecdysteroids in the total radioactive materials decreased at first and increased subsequently. This change from a decrease to a increase in the unconjugated ecdysteroids suggested the possibility of a release of the unconjugated ecdysteroids from the conjugated ecdysteroids, especially from OA. HPLC analysis also revealed that the ecdysteroids released from the conjugate were mainly 20-hydroxyecdysone. Therefore, we propose a scheme as shown in Fig. 9, for the inactivation and reactivation process of the moulting hormone at the stage from pupa to pharate adult in $S$. peregrina. The conjugate $\mathrm{OB}$ injected into the pharate pupae did not change to other metabolites. Whereas, the injected $\mathrm{OA}$ was converted to $\mathrm{OB}$ and free ecdysteroids. It can be assumed, therefore, that $\mathrm{OB}$ may be the final inactivated substance of 20-hydroxyecdysone, and that $\mathrm{OA}$ may be a specific inactivated substance from which 20 -hydroxyecdysone can be released. In other words, the process by which 20 -hydroxyecdysone was metabolized to $\mathrm{OA}, \mathrm{OB}$ and the unknown ecdysteroids is regarded as an inactivation process, and the release of 20-hydroxyecdysone from OA may be involved in the possible reactivation process of the moulting hormone. It is not clear, however, whether or not $\mathrm{OB}$ is synthesized from OA.

It is interesting to us that the percentage of radioactive 20-hydroxyecdysone to the total metabolites increased 2 days after the injection, namely at the stage corresponding to the onset of adult development. These facts suggest that ecdysteroid conjugate $\mathrm{OA}$, which is synthesized as an inactivated product at the stage of larval-pupal development and may be hydrolyzed at the stage of pupal-adult development to liberate 20-hydroxyecdysone, would be, at least in part, used for adult development.

Ecdysteroid conjugates have been detected in various insect species, includ- 
ing Bombyx mori (Moriyama et al., 1970; Mizuno and Ohnishi, 1975), Calliphora erythrocephala (Shaaya and Karlson, 1965; Heinrich and Hoffmeister, 1970), Antheraea polyphemus (Cherbas and Cherbas, 1970), Locusta migratoria (Koolman et al., 1973), Choristoneura fumiferena (Lagueux et al., 1976), Schistocerca gregaria (Wilson and Morgan, 1978). Gryllus bimaculatus (Wildmann and Romer, 1977) and Galleria mellonella (Maróy and Tarnóy, 1978). Recently, changes in the ecdysteroid titer during particular developmental stages of arthoropodes were reported, and formation of ecdysteroid conjugates was also described (McCarthy and Shimmer, 1979; Hsiao and Hsiao, 1979; Gande and Morgan, 1979).

Lagueux and co-workers (1979) mentioned that the decrease in the peak concentration of moulting hormone was achieved essentially through conjugation. Gande et al. (1979) also documented that during embryonic development the pattern changes from a high proportion of conjugated ecdysteroids in the early stages to a higher proportion of 20 -hydroxyecdysone in the later stages.

We claim that during the postembryonic development a biological balance between free hormone and conjugated hormone is a very important factor for leading the insects to normal metamorphosis. Chemical identification of these conjugates and the unknown ecdysteroids is now in progress.

\section{ACKNOWLEDGEMENTS}

We wish to express our appreciation to Dr. S. Nakazawa, Laboratory of Technology, and Dr. Y. Akamatsu, Department of Chemistry, for their useful advice during this work. We also thank Dr. S. Kawabata, Department of Biomedical Research on Foods, and Dr. E. Okada, Tokyo Research and Applications Laboratory, Shimadzu Corp., for their helpful instruction in performing HPLC.

\section{REFERENCES}

Cherbas, L. And Cherbas, P. (1970): Distribution and metabolism of $\alpha$-ecdysone in pupae of the silkworm Antheraeae polyphemus. Biol. Bull., Woods Hole, 138, 115-128.

Gande, A. R. And Morgan, E. D. (1979): Ecdysteroids in the developing eggs of the desert locust, Schistocerca gregaria. J. Insect Physiol., 25, 289-293.

Gande, A. R., Morgan, E. D. AND Wilson, I. D. (1979): Ecdysteroid levels throughout the life cycle of the Desert Locust, Schistocerca gregaria. J. Insect Physiol., 25, 669-675.

Hsiao, T. H. And Hsiao, C. (1978): Ecdysteroids in the ovary and the egg of the greater wax moth. J. Insect Physiol., 25, 45-52.

Heinrich, G. AND Hoffmeister, H. (1970): Bildung von Hormonglykosidasen als Inaktivierungsmechamismus bei Calliphora erythrocephala. Z. Naturforsch., 25b, 358-361.

Koolman, J., Hoffmann, J. A. and Karlson, P. (1973): Sulphate esters as inactivation products of ecdysone in Locusta migratoria. Hoppe-Seyler's Physiol. Chem., 354, 1043-1048.

Lagueux, M., Hetru, C., Goltzene, F., Kappler, C. and Hoffmann, J. A. (1979): Ecdysone titre and metabolism in relation to cuticulogenesis in embryos of Locusta migratoria. J. Insect Physiol., 25, 709-723.

Lagueux, M., Perron, J. M. and Hoffmann, J. A. (1976): Ecdysone metabolism and endogenous moulting hormone titer during larval-pupal development in Choristoneura fumiferana. J. Insect Physiol., 22, 57-62.

Maróy, P. AND TARNóy, K. (1978): Moulting hormone level during the last instar of Galleria mellonella larva. J. Insect Physiol., 24, 325-327. 
Mizuno, T. And OHnishi, E. (1975): Conjugated ecdysone in the eggs of the silkworm, Bombyx mori. Develop. Growth Differ., 17, 219-225.

McCarthy, J. F. ANd Shinner, D. M. (1979): Changes in ecdysteroids during embryogenesis of the blue crab, Callinectes sapidus Rathlum. Develop. Biol., 69, 627-633.

Moribayashi, A. F. AND OHTAKI, T. (1978): Inactivation of ecdysone and the possible feed back control of the titre during pupation of Sarcophaga peregrina. J. Insect Physiol., 24, 279-284.

Moriyama, H., Nakanishi, K., King, D. S., Okauchi, T., Siddall, J. B. and Hafferl, W. (1970): On the origin and metabolic fate of $\alpha$-ecdysone in insects. Gen. Comp. Endocr., 15, 80-87.

Ohtaki, T., Milkman, R. D. and Williams, C. M. (1968): Dynamics of ecdysone secretion and action in the fleshfly Sarcophaga peregrina. Biol. Bull., Woods Hole, 135, 322-334.

OHTAKI, T. AND TAKAHASHI, M. (1972): Introduction and termination of pupal diapause in relation to the change of ecdysone titer in the fleshfly, Sarcophaga peregrina. Japan. J. Med. Sci. Biol., 25, 369-376.

ShaAya, E. AND KarLson, P. (1965): Der Ecdysontiter wahrend der Insektenetruicklung. II. Die postembryonale Entwicklung der Schmeissfliege Calliphora erythocephala Meig. J. Insect Physiol., 11, 65-69.

WildMANN, H. AND Romer, F. (1977): Metabolism of ${ }^{3} \mathrm{H}-\alpha$-ecdysone in Gryllus bimaculatus during the eighth larval instar. J. Insect Physiol., 23, 343-349.

WiLson, I. D. AND MoRgAn, E. D. (1978): Variation in ecdysteroid levels in 5th instar larvae of Schistocerca gregaria in gregarians and solitary phases. J. Insect Physiol., 24, 751-756. 